\title{
Resource utilization and cost saving analysis of subcutaneous versus intravenous trastuzumab in early breast cancer patients
}

\author{
Alberto Farolfi ${ }^{1}$, Paolo Silimbani ${ }^{2}$, Davide Gallegati ${ }^{3}$, Elisabetta Petracci ${ }^{4}$, Alessio \\ Schirone $^{1}$, Mattia Altini ${ }^{5}$ and Carla Masini ${ }^{2}$ \\ ${ }^{1}$ Department of Medical Oncology, Istituto Scientifico Romagnolo per lo Studio e la Cura dei Tumori (IRST) IRCCS, Meldola, Italy \\ ${ }^{2}$ Oncology Pharmacy Unit, Istituto Scientifico Romagnolo per lo Studio e la Cura dei Tumori (IRST) IRCCS, Meldola, Italy \\ ${ }^{3}$ Financial and Management Control, Istituto Scientifico Romagnolo per lo Studio e la Cura dei Tumori (IRST) IRCCS, Meldola, Italy \\ ${ }^{4}$ Unit of Biostatistics and Clinical Trials, Istituto Scientifico Romagnolo per lo Studio e la Cura dei Tumori (IRST) IRCCS, Meldola, Italy \\ ${ }^{5}$ Healthcare Administration, Istituto Scientifico Romagnolo per lo Studio e la Cura dei Tumori (IRST) IRCCS, Meldola, Italy
}

Correspondence to: Alberto Farolfi, email: alberto.farolfi@irst.emr.it

Keywords: breast cancer, economic evaluation, oncology, subcutaneous trastuzumab

Received: February 07, $2017 \quad$ Accepted: June 02, $2017 \quad$ Published: June 16, 2017

Copyright: Farolfi et al. This is an open-access article distributed under the terms of the Creative Commons Attribution License 3.0 (CC BY 3.0), which permits unrestricted use, distribution, and reproduction in any medium, provided the original author and source are credited.

\section{ABSTRACT}

We conducted an economic evaluation of intravenous (IV) vs subcutaneous (SC) trastuzumab for the treatment of patients with early breast cancer (EBC). Data of patients receiving adjuvant IV trastuzumab at our institute in 2014 were used to study three different treatment scenarios: 1) IV trastuzumab, 2) SC trastuzumab, and 3) IV trastuzumab during chemotherapy followed by SC trastuzumab. Our cohort included 114 patients with a median weight of $63.75 \mathrm{~kg}$. Scenario 2 was the most time-saving treatment, with $71.7 \%$ reduction in preparation time and $89.3 \%$ reduction in chair time compared to scenario 1 . Considering full costs, the mean costs per patient/year were $\subset 14,233 \pm 8,698$ for scenario $1, C 14,272 \pm 8,312$ for scenario 2 , and $C 14,535$ $\pm 8,646$ for scenario $3(p=0.959)$. When mean body weight was $>65.2 \mathrm{~kg}$, the mean cost was lower in scenario 2 than in scenario 1 . Scenario 2 proved a valuable time-saving and cost-saving option. A shift from IV to SC trastuzumab should be considered, especially in capacity-constrained oncology departments.

\section{INTRODUCTION}

Humanized monoclonal antibody trastuzumab has been shown to have a significant survival benefit for patients with early breast cancer (EBC) [1]. Until a few years ago, trastuzumab was available only as an intravenous (IV) infusion, whose dose was calculated according to the patient's body weight. The loading dose was administered for at least 90 minutes followed by a maintenance dose of at least 30 minutes [2]. The subcutaneous (SC) formulation administered via hand-held syringe contained a fixed dose of $600 \mathrm{mg}$ trastuzumab and the excipient recombinant human hyaluronidase (rHuPH20). It has sought approval from the European Medicines Agency for EBC as an alternative to the conventional IV trastuzumab, based on comparable pharmacokinetics and efficacy results, and similar safety profiles provided by a non-inferiority study [3]. SC trastuzumab has proved superior over IV according to patient preference, because it saved time (administered for 5 minutes without any need of a loading dose), causing less pain, discomfort and side-effects [4]. SC administration results in a faster and equally safe method of treatment delivery, enabling physicians and nurses to save time and increase the number of patients that can be treated during their working hours [5].

Nevertheless, both formulations must be adequately prepared before administration. Manual preparation of cytotoxic drugs has always been considered a high-risk procedure, given the prolonged exposure to carcinogens during handling [6] and the high level of therapy personalization, which leads to greater chances of error [7].

The introduction of robots in the drug preparation process has aimed to reduce both the number of errors 
and the risk for the staff from exposure to carcinogenic agents, improving product quality in terms of drug dosage accuracy and sterility. In our previous study on the quality and economic implications of manual vs automated preparation of antineoplastic drugs, including trastuzumab, we demonstrated that both procedures were accurate. The automated preparation yielded better quality maintenance standards (i.e. higher accuracy of the active ingredient concentration) and lower risk for operators [8]. Manual dosing may lead to occasional errors which are difficult to track down: we found that manual preparation resulted in $>10 \%$ discrepancy between prescribed and prepared dose of trastuzumab [8].

The results of the HannaH and PrefHer studies demonstrated that SC trastuzumab is an efficacious, welltolerated treatment, and a valuable option for patients and healthcare professionals. However, concerns about increasing costs of the fixed dose of SC trastuzumab are limiting its use. Basing our investigation on the Italian Health System key principles of equitable and sustainable care, we evaluated the resource utilization associated with the administration of SC trastuzumab compared with IV trastuzumab in patients with HER2-positive EBC. Advanced settings were not considered, as an IV trastuzumab biosimilar $[9,10]$, whose direct costs are still unknown, will soon be available.

We analyzed direct and indirect costs associated with both formulations in the adjuvant setting in 3 scenarios in order to determine the most performing in terms of cost saving: IV trastuzumab (scenario 1), SC trastuzumab (scenario 2), and IV trastuzumab during chemotherapy followed by SC trastuzumab after chemotherapy (scenario 3). Scenario 3 was designed considering that the PrefHer trial showed that the safety profile was not affected in any way by the switch from SC to IV or viceversa [11].

\section{RESULTS}

\section{Direct costs}

In 2014, 114 breast cancer patients with a median weight of $63.75 \mathrm{~kg}$ (range 55-74.9) were treated with adjuvant IV trastuzumab. Table 1 shows the main patient characteristics. Considering the adverse events, 4 patients (3.5\%) prematurely interrupted the treatment because of cardiotoxicity (asymptomatic left ventricular ejection fraction drop). Normal heart function was restored once treatment had been discontinued without sequelae. Other five patients $(4.38 \%)$ experienced a significant reduction in left ventricular ejection fraction, without the need of treatment interruption. Five patients $(4.38 \%)$ had relapsed by the time of the analyses.

IV trastuzumab was delivered in 1,292 cycles, corresponding to 937 cycles of SC trastuzumab in scenario 2 , and 543 cycles of IV trastuzumab, and 749 cycles of
SC trastuzumab in scenario 3. Overall, 372,214 mg IV trastuzumab were administered, corresponding to 562,200 $\mathrm{mg}$ SC trastuzumab in scenario 2, with a difference of $189,986 \mathrm{mg}$ in drug dosage $(\Delta=51.0 \%$ in trastuzumab dose) (Table 2). The total cost of the drugs were as follows: $€ 1,544,688$ for scenario $1, € 1,613,514$ for scenario 2 , and $€ 1,612,275$ for scenario 3 , for a total of $77,710 \mathrm{mg}$ IV trastuzumab delivered in 543 cycles and $449,400 \mathrm{mg}$ SC trastuzumab in 749 cycles. Given an average of $0.11 \%$ drug waste of trastuzumab during preparation, regardless of the days in which most costly therapies are prepared, the cost of drug waste amounted to $€ 1,699$ and $€ 344$ for scenarios 1 and 3, respectively. Consequently, the mean direct costs per patient were: $€ 13,655 \pm 8,412$, $€ 14,154$ $\pm 8,243$, and $€ 14,146 \pm 8,514$ for scenario 1,2 and 3 , respectively. Differences in direct costs among the three scenarios were not statistically significant $(p=0.832)$.

\section{Indirect costs}

$\mathrm{SC}$ trastuzumab required an average preparation time $24.1 \%$ shorter than IV trastuzumab per cycle. Given the lower number of preparations in scenario 2, the overall preparation time was $71.7 \%$ shorter for SC trastuzumab. Mean preparation time per patient was $10.5 \pm 6.3$ hours, $3.0 \pm 1.7$ hours, and $3.7 \pm 5.2$ hours for scenarios 1,2 and 3 , respectively. This difference was statistically significant, $\mathrm{p}_{\text {omnibus }}<0.001$ ( $\mathrm{p}$ for scenario 2 vs $1<0.001$, $\mathrm{p}$ for scenario 3 vs $1<0.001$, and p for scenario 3 vs $2<0.001$ ) (Table 3 ).

Administration of SC trastuzumab in scenario 2 reduced nursing and chair time by an average of $89.3 \%$ compared with IV trastuzumab. In scenario 3, the overall time of administration was reduced by $47 \%$ (Table 3 ). Mean administration time per patient was $6.4 \pm 3.7$ hours, $0.7 \pm 0.4$ hours and $3 \pm 3.2$ hours for scenarios 1,2 and 3 , respectively. Again, these differences were statistically significant, $\mathrm{p}_{\text {omnibus }}<0.001$ ( $\mathrm{p}$ for scenario 2 vs $1<0.001$, $\mathrm{p}$ for scenario 3 vs $1<0.001$, and $\mathrm{p}$ for scenario 3 vs 2 $<0.001)$.

The preparation of one cycle of IV trastuzumab cost $€ 8.17$, whereas one cycle of SC trastuzumab $€$ 6.99. Mean preparation costs per patient were $€ 92.60$ $\pm 55.80, € 57.50 \pm 33.50$, and $€ 84.80 \pm 52.70$. This led to a statistically significant difference among the three scenarios, $\mathrm{p}_{\text {omnibus }}<0.001$ considering scenario 2 vs scenario $1(€-35.14, p<0.001)$, scenario 3 vs scenario 2 (€ -27.39, $p<0.001)$. This was not the case between scenarios 3 and $1(€-7.75, p=0.681)$.

Occupational costs of outpatient clinic were different across scenarios: $€ 65,644$ for scenario $1, € 7,012$ for scenario 2, and $€ 34,753$ for scenario 3 (Table 4). Mean outpatient clinic costs per patient were: $€ 575.82 \pm 329.15$, $€ 61.51 \pm 35.82$ and $€ 304.78 \pm 284.06$ for scenarios 1,2 and 3 respectively, $\mathrm{p}_{\text {omnibus }}<0.001$ ( $\mathrm{p}$ for scenario 2 vs 1 $<0.001$, p for scenario 3 vs $1<0.001$, and $\mathrm{p}$ for scenario 3 vs $2<0.001)$. 
Table 1: Patient characteristics

\begin{tabular}{|c|c|c|}
\hline & No. & $(\%)$ \\
\hline Median age, years [range] & 56 & [33-82] \\
\hline Median weight, kg [range] & 63.75 & [42-95] \\
\hline $\begin{array}{l}\text { Histology } \\
\text { Ductal } \\
\text { Lobular } \\
\text { Other }\end{array}$ & $\begin{array}{c}110 \\
2 \\
2\end{array}$ & $\begin{array}{l}(96.5) \\
(1.75) \\
(1.75)\end{array}$ \\
\hline $\begin{array}{l}\text { Nuclear grade } \\
\text { G1 } \\
\text { G2 } \\
\text { G3 } \\
\end{array}$ & $\begin{array}{c}0 \\
28 \\
86 \\
\end{array}$ & $\begin{array}{c}- \\
(24.6) \\
(75.4) \\
\end{array}$ \\
\hline $\begin{array}{l}\text { ER status } \\
\quad \geq 1 \% \\
\quad<1 \%\end{array}$ & $\begin{array}{l}80 \\
34\end{array}$ & $\begin{array}{l}(70.2) \\
(29.8)\end{array}$ \\
\hline $\begin{array}{l}\text { PgR status } \\
\quad \geq 1 \% \\
\quad<1 \%\end{array}$ & $\begin{array}{l}63 \\
51\end{array}$ & $\begin{array}{l}(55.3) \\
(44.7)\end{array}$ \\
\hline $\begin{array}{l}\text { Ki67 } \\
\quad \text { High }(\geq 20 \%) \\
\text { Low }(<20 \%)\end{array}$ & $\begin{array}{l}16 \\
98\end{array}$ & $\begin{array}{l}(14) \\
(86)\end{array}$ \\
\hline $\begin{array}{c}\text { Tumor stage } \\
\text { T1 } \\
\text { T2 } \\
\text { T3 } \\
\text { T4 }\end{array}$ & $\begin{array}{c}64 \\
42 \\
5 \\
3\end{array}$ & $\begin{array}{l}(56.2) \\
(36.8) \\
(4.4) \\
(2.6)\end{array}$ \\
\hline $\begin{array}{c}\text { Nodal stage } \\
\text { N0 } \\
\text { N1 } \\
\text { N2 } \\
\text { N3 } \\
\end{array}$ & $\begin{array}{c}69 \\
32 \\
8 \\
5\end{array}$ & $\begin{array}{c}(60.5) \\
(28.1) \\
(7) \\
(4.4) \\
\end{array}$ \\
\hline $\begin{array}{l}\text { Type of chemotherapy } \\
\text { Adjuvant } \\
\text { Neoadjuvant } \\
\end{array}$ & $\begin{array}{l}96 \\
18 \\
\end{array}$ & $\begin{array}{l}(84.2) \\
(15.8)\end{array}$ \\
\hline $\begin{array}{l}\text { Chemotherapy scheme } \\
\text { AC or EC } \\
\text { AC or EC followed by wP } \\
\text { FEC followed by wP or D } \\
\text { wP } \\
\text { DC } \\
\text { TCH } \\
\text { CMF } \\
\text { Vinorelbine }\end{array}$ & $\begin{array}{c}5 \\
64 \\
21 \\
11 \\
3 \\
3 \\
4 \\
3\end{array}$ & $\begin{array}{c}(4.4) \\
(56.2) \\
(18.4) \\
(9.7) \\
(2.6) \\
(2.6) \\
(3.5) \\
(2.6)\end{array}$ \\
\hline
\end{tabular}

Abbreviations: AC, adriamycin and cyclophosphamide; CMF, cyclophosphamide, methotrexate and fluorouracil; EC, epirubicin and cyclophosphamide; D, docetaxel; DC, docetaxel and cyclophosphamide; ER, estrogen receptor; FEC, fluorouracil epirubicin and cyclophosphamide; PgR, progesterone receptor; TCH, docetaxel, carboplatin and trastuzumab; wP, weekly paclitaxel.

\section{Cost per patient}

Considering the direct and indirect costs between scenarios, we calculated an overall cost of $€ 1,899,217$ for scenario $1, € 1,902,374$ for scenario 2 , and $€ 1,933,681$ for scenario 3, which equates to a mean cost per patient of $€ 14,233.20 \pm 8,698.41$ for scenario $1, € 14,272.60 \pm$ $8,312.63$ for scenario 2 , and $€ 14,535.30 \pm 8,646.76$ for scenario 3. Mean global costs per patient among the three scenarios were not statistically significant $(p=0.959)$. 
Table 2: Weight quartiles of patient population and trastuzumab in the two formulation doses infused per quartile

\begin{tabular}{|l|c|c|c|}
\hline \multicolumn{1}{c|}{ Weight quartiles } & IV trastuzumab dose $(\mathbf{m g})$ & SC trastuzumab dose (mg) & $\boldsymbol{\Delta}(\mathbf{m g})$ \\
\hline $\mathrm{Q} 1=55 \mathrm{~kg}$ & 78,138 & 154,800 & 76,662 \\
\hline $\mathrm{Q} 2=63.7 \mathrm{~kg}$ & 82,938 & 133,800 & 50,862 \\
\hline $\mathrm{Q} 3=74.9 \mathrm{~kg}$ & 107,514 & 152,400 & 44,886 \\
\hline $\mathrm{Q} 4=95 \mathrm{~kg}$ & 103,624 & 121,200 & 17,576 \\
\hline Total & 372,214 & 562,200 & 189,986 \\
\hline
\end{tabular}

Q, quartile; IV, intravenous; SC, subcutaneous

Table 3: Time per preparation and administration of trastuzumab in the three scenarios

\begin{tabular}{|l|c|c|c|c|c|}
\hline \multicolumn{2}{c}{ Scenario 1 } & \multicolumn{1}{c}{ Scenario 2 } & $\boldsymbol{\Delta}(\mathbf{\%})$ & Scenario 3 & $\boldsymbol{\Delta}(\mathbf{\%})$ \\
\hline Unit preparation time (sec) & 844 & 641 & $-203(24.1)$ & - & - \\
\hline Overall preparation time (h) & 120 & 34 & $-86(71.7)$ & 78 & $-42(35)$ \\
\hline Mean preparation time per patient (h) & $10.5 \pm 6.3$ & $3.0 \pm 1.7$ & $-7.2(71.7)$ & $6.8 \pm 5.2$ & $-3.7(35)$ \\
\hline Unit administration time (min) & & & & & \\
$\quad$ Loading dose [No. of cycles] & $90[85]$ & $5[85]$ & $-85(94.4)$ & $90[53]^{*} ; 5[32]$ & 0 \\
$\quad$ Maintenance dose [No. of cycles] & $30[1,207]$ & $5[1,212]$ & $-25(83.3)$ & $30[490] ; 5[717]$ & \\
\hline Overall administration time (h) & 731 & 78 & $-653(89.3)$ & -387 & $-344(47)$ \\
\hline Mean administration time per patient (h) & $6.4 \pm 3.7$ & $0.7 \pm 0.4$ & $-5.7(89.3)$ & $3.4 \pm 3.2$ & $-3.0(47)$ \\
\hline
\end{tabular}

sec, seconds; h, hours; min, minutes

*The number of loading doses is lower than in scenario 1 because 32 patients received trastuzumab for the first time after chemotherapy and were thus directly "transferred" to subcutaneous trastuzumab.

We subsequently designed a model considering all the costs associated with trastuzumab taking weight as a variable, only for scenarios 1 and 2 , since scenario 3 proved non-performing for economical evaluation. The model in Figure 1 shows that if the mean weight of patients treated with adjuvant trastuzumab is $>65.2 \mathrm{~kg}$ (break even point), scenario 2 will be less expensive than scenario 1 .

\section{DISCUSSION}

SC trastuzumab proved to be non-inferior to IV trastuzumab in terms of event-free survival and overall survival in a large randomized trial with a median follow-up of over 40 months, with no difference in the frequency and/or severity of adverse events despite the different dosages of the formulations [12]. Considering the patients' preference of SC over IV trastuzumab [4] and the saving of preparation and administration times, the SC formulation should be considered as a valuable clinical opportunity to improve the efficiency of HER2-positive breast cancer treatment delivery.

Our analysis was conducted to evaluate the economic implications of this new formulation with an innovative approach, which considered not only direct drug costs but also indirect costs. This led to the hypothesis of a switch in drug formulation from
IV to SC. To this aim, we built three scenarios for the adjuvant treatment of HER2-positive EBC: 1) standard of care with the IV formulation; 2) SC trastuzumab only; 3) IV trastuzumab during chemotherapy followed by SC trastuzumab, in accordance with the PreHer trial, which showed no difference in safety for the prescribed sequence [11].

Even if mean global costs per patient were not statistically different, scenario 3 was shown to be the worst in terms of cost saving with a mean cost per patient/ year of $€ 14,535$. This result was against our expectations, as the indirect costs were much higher than in scenario 2 . Scenario 2 was slightly more expensive than scenario 1 in drug cost per patient (i.e. $€ 39.40$ per patient/year), yet less expensive in terms of cost of time (i.e. opportunity cost): staff and other resources could be directed to other activities, i.e. patient management and treatment delivery. In addition, scenario 2 resulted more advantageous in terms of "value" (i.e. health and quality outcome for money spent), with benefits of patient time saving. Compared to scenario 1 , the higher overall cost of scenario 2 lowered with the increase in the average patient body weight, resulting equal in cost (break even point) when mean body weight was $>65.2 \mathrm{~kg}$ (Figure 1).

As a previous study [8] demonstrated that the average preparation unit cost in terms of labor and waste was lower in automated than in manual procedures, we hypothesized 
Table 4: Mean cost per patient among the three scenarios

\begin{tabular}{|l|c|c|c|c|}
\hline \multicolumn{2}{c}{ Scenario 1 } & Scenario 2 & \multicolumn{3}{c}{ Scenario 3 } \\
\hline Direct cost & $13,655 \pm 8,412$ & $14,154 \pm 8,243$ & $14,146 \pm 8,514$ & 0.832 \\
\hline Preparation cost & $92.6 \pm 55.8$ & $57.5 \pm 33.5$ & $84.8 \pm 52.7$ & $<0.001$ \\
\hline Day hospital costs & $575.82 \pm 329.15$ & $61.51 \pm 35.82$ & $304.78 \pm 284.06$ & $<0.001$ \\
\hline Global cost & $14,233.2 \pm 8,698.41$ & $14,272.6 \pm 8,312.63$ & $14,535.3 \pm 8,646.76$ & 0.959 \\
\hline
\end{tabular}

a totally robotic drug preparation. Differences in resource utilization could therefore be less marked where manual preparation is still a standard of care.

In our study population, median body weight was $63.75 \mathrm{~kg}$, whereas in the PrefHer trial was $68 \mathrm{~kg}$ and $66 \mathrm{~kg}$ in arms A and B, respectively [13]. Given that in the HannaH trial no difference in adverse events or pharmacokinetics was reported with respect to body weight [3], our evaluation supports even more the rationale for the choice of SC over IV trastuzumab, not to mention patient preference. The higher direct costs of SC trastuzumab are counterbalanced by the reduced resource utilization related to the time saved in drug preparation and administration.

These results are in line with the economical evaluation of a similar context in New Zealand: the switch

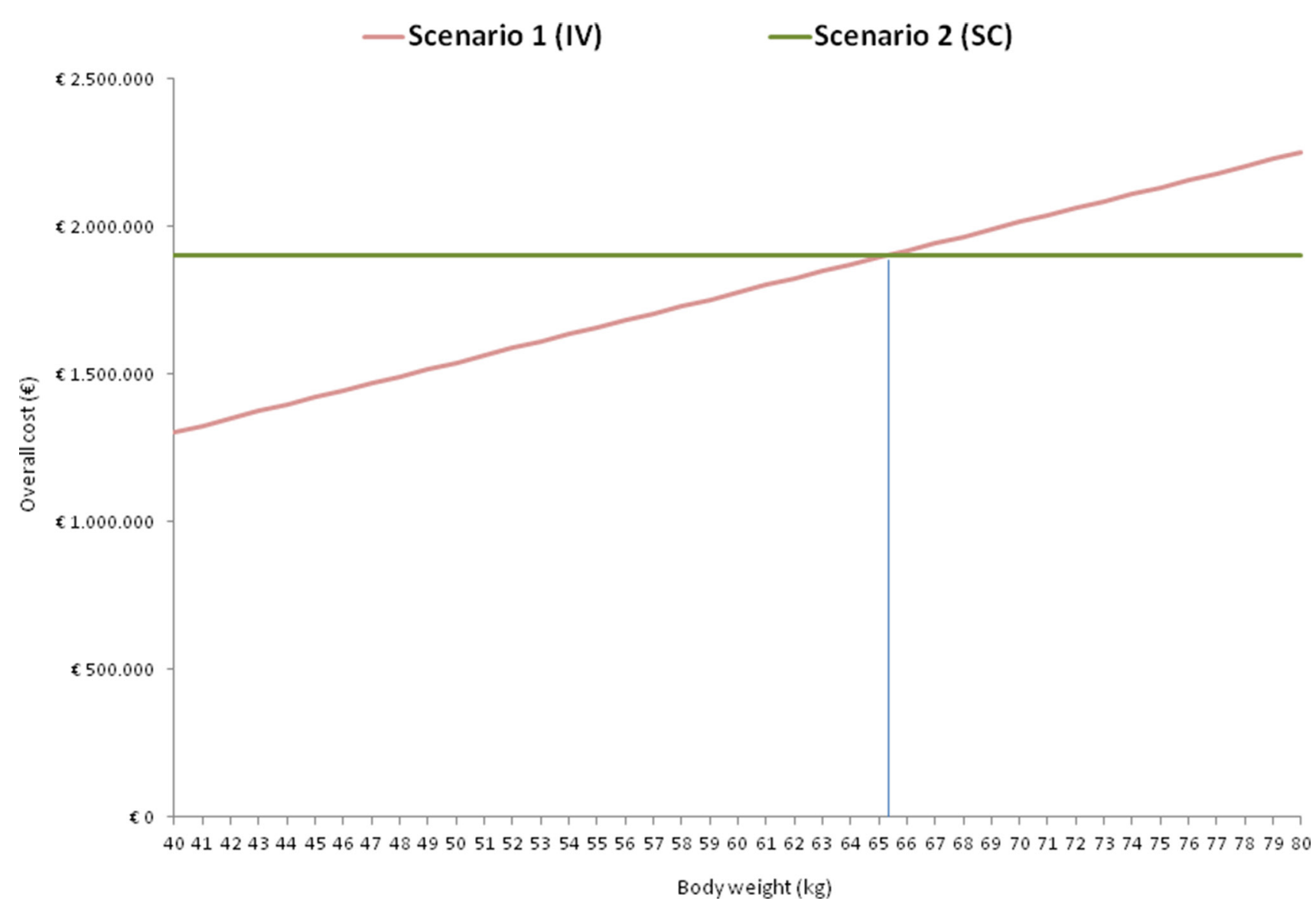

Figure 1: Overall cost per scenario according to body weight in scenarios 1 and 2. between formulations reduced both the time spent in the clinic and the healthcare professional resources and consumables needed for administration, contributing to an overall reduction in healthcare costs [14]. As in the PrefHer trial, SC trastuzumab showed to save more patient chair and healthcare professional time than IV infusion [15].

Healthcare decision makers should consider that a change in the trastuzumab formulation from IV to SC will lead to a considerable, immediate rise in drug cost, yet counterbalanced by other economic advantages, such as the possibility to treat a greater number of patients, improving hospital accessibility, reducing waiting lists while maintaining standards of care, and increasing treatment capacity by relieving capacity-constrained oncology departments. Hospitals with acceptable and manageable waiting lists and on lower budgets may find 
the use of SC trastuzumab inconveniently costly. Patient time-saving benefits from SC trastuzumab preparation and administration should also be taken into account.

This study has a few limitations that must be highlighted. First, the results of this study may not be generalized due to the different direct and indirect drug costs across countries. It can be hypothesized that scenario 3 will be the worst scenario, regardless of the actual costs. Patient preference, well-being, and quality of life were not assessed in this study, although discussed in the PrefHer study [4, 13]. By far the most common reason for patients to prefer SC trastuzumab was that it saved time [13], as our study shows. Moreover, the impact and the costs of indwelling IV catheter insertion, maintenance, and complication-related management were not included in the analysis. Finally, although we designed three different scenarios starting from a real-life situation (our 2014 experience), the scenarios might detach from the actual situation, in particular with respect to the number of patients treated and their mean body weight. To reach more generalizable findings, we reported the mean cost per patient/year and created a model with body weight as a variable.

\section{MATERIALS AND METHODS}

In order to compare the resource utilization of the two trastuzumab formulations, we designed a simple model including all the patients treated with adjuvant trastuzumab whose data were retrieved from the institutional medical record database ( $\log 80)$ from January 1st, 2014 to December 31st, 2014. The database collects all the pharmacy and medical data of the patients treated at the Istituto Scientifico Romagnolo per lo Studio e la Cura dei Tumori (IRST) IRCCS (Meldola, Italy). On the basis of this retrospective cohort study, we simulated that the same cohort would be treated with either IV or SC trastuzumab for the whole time or with IV trastuzumab during chemotherapy followed by SC trastuzumab.

Based on a previous work [8], we hypothesized that both formulations would be robotically prepared.

\section{Robotic drug preparation}

The robot $\left(\right.$ ApotecaCHEMO $\left.{ }^{\circledR}\right)$ is located in a closed, microbiologically controlled environment. Its anthropomorphic arm mechanically replicates the actions of a human operator. The system allows for continuous checks on the entire preparation process. All of the production steps, as well as incoming and outgoing materials, are checked and recorded by technological devices, such as sensors, photocells, a vision system, and barcode readers. Automatic identification of drugs, weight-checking system, and barcode labeling guarantee process traceability. Preparation time was calculated as the time between the first and last weighing of components.

\section{Resource utilization analysis}

For each hypothesized model, we calculated: a) the direct cost of the drug used (only one type of trastuzumab is commercially available in Italy - Herceptin ${ }^{\circledR} 150$ $\mathrm{mg}$, Roche): $€ 4.15 / \mathrm{mg}$ for IV formulation and $€ 2.87 /$ $\mathrm{mg}$ for SC formulation; b) the differential gap $(\Delta)$ of milligrams of drug administered between the two different formulations; c) drug preparation time and unit costs for a healthcare professional; and d) drug administration time and unit costs for outpatient clinic management per hour. As patients may have received $2 \mathrm{mg} / \mathrm{kg}$ IV trastuzumab weekly during chemotherapy, 3 preparations were converted into one of SC trastuzumab administered every 3 weeks, maintaining preparation and administration times separate.

Costs for indwelling venous lines (e.g. central venous catheter - Portacath and Groshong) were not considered as patients had already had them fixed for previous chemotherapy. Unit costs for healthcare professionals were retrieved from the Italian National Contract, and were as follows: $€ 21$ /hour for a technician and $€ 60$ /hour for a hospital pharmacist. Unit costs for outpatient clinic management was $€ 89.80$ /hour retrieved from the personnel costs, resource utilization (excluding drug costs) and management cost for the total number of hours of outpatient clinic use.

For both formulations, the average time for preparation did not include the time for the transfer of supplies from the storage room to the pharmacy, the prelabeling of the input material and the time for dressing change of the operators. The average preparation time was therefore calculated from the actual start time of reconstitution of drug vials and preparation of bags, labeling and identification of the preparation by means of a barcode scan.

\section{Statistical analyses}

Descriptive statistics are reported as frequencies and percentages for categorical variables and as mean and standard deviation (SD) or median and range for continuous variables. The analysis of variance (ANOVA) was performed to compare the means among the three scenarios. Post-hoc comparisons were performed when the omnibus F-test was statistically significant. The Bonferroni method was used to adjust for multiple comparisons. A two-sided $p$-value $<0.05$ was deemed statistically significant for all the analyses. All statistical analyses were carried out using STATA 10.1 statistical software (StataCorp, College Station, TX, USA).

\section{CONCLUSIONS}

Data from our study demonstrated that treatment with SC trastuzumab should be initiated immediately, 
without switching from another treatment. SC trastuzumab proved a time-saving and a potential cost-saving treatment without significantly increasing the mean global costs per patient. This resulted in a reduction in resource utilization and an improvement of the capacity of oncology departments to treat a higher number of patients. We showed that economic evaluations of drugs should not only include the direct costs, but also the consequences of the different treatment modalities, i.e. indirect costs.

\section{Authors' contributions}

CM conceived the idea for the project and was project leader. AF and PS performed data analyses and drafted the manuscript. DG assisted with data analysis. MA and AS reviewed the manuscript for important intellectual content. All authors approved the final version of the manuscript.

\section{ACKNOWLEDGMENTS}

The authors wish to thank Veronica Zanoni and Cristiano Verna for editing the manuscript.

\section{CONFLICTS OF INTEREST}

The authors have no competing interests to declare.

\section{FINANCIAL SUPPORT}

No funding was received.

\section{REFERENCES}

1. Dahabreh IJ, Linardou H, Siannis F, Fountzilas G, Murray S. Trastuzumab in the adjuvant treatment of early-stage breast cancer: a systematic review and metaanalysis of randomized controlled trials. Oncologist. 2008; 13:620-630.

2. European Medicines Agency. Herceptin. Summary of product characteristics. http://www.ema.europa.eu/docs/ en_GB/document_library/EPAR [Accessed 15 June 2016] .

3. Jackisch C, Kim SB, Semiglazov V, Melichar B, Pivot X, Hillenbach C, Stroyakovskiy D, Lum BL, Elliott R. Subcutaneous versus intravenous formulation of trastuzumab for HER2-positive early breast cancer: updated results from the phase III HannaH study. Ann Oncol. 2015; 26:320-325.

4. Pivot X, Gligorov J, Müller V, Barrett-Lee P, Verma S, Knoop A, Curigliano G, Semiglazov V, López-Vivanco G, Jenkins V, Scotto N, Osborne S, Fallowfield L. Preference for subcutaneous or intravenous administration of trastuzumab in patients with HER2-positive early breast cancer (PrefHer): an open-label randomised study. Lancet Oncol. 2013; 14:962-970.
5. Rule S, Collins GP, Samanta K. Subcutaneous vs intravenous rituximab in patients with non-Hodgkin lymphoma: a time and motion study in the United Kingdom. J Med Econ. 2014; 17:459-468.

6. Connor TH, DeBord DG, Pretty JR, Oliver MS, Roth TS, Lees PS, Krieg EF Jr, Rogers B, Escalante CP, Toennis CA, Clark JC, Johnson BC, McDiarmid MA. Evaluation of antineoplastic drug exposure of health care workers at three university-based US cancer centers. J Occup Environ Med. 2012; 52:1019-1027.

7. Bonan B, Martelli N, Berhoune M, Maestroni ML, Havard L, Prognon P. The application of hazard analysis and critical control points and risk management in the preparation of anti-cancer drugs. Int J Qual Health Care. 2009; 21:44-50.

8. Masini C, Nanni O, Antaridi S, Gallegati D, Marri M, Paolucci D, Minguzzi M, Altini M. Automated preparation of chemotherapy: quality improvement and economic sustainability. Am J Health Syst Pharm. 2014; 71:579-585.

9. Rugo HS, Barve A, Waller CF. Heritage: a phase III safety and efficacy trial of the proposed trastuzumab biosimilar Myl-1401O versus Herceptin. J Clin Oncol. 2016; 34.

10. Shustova M, Burdaeva O, Alexeev S. Efficacy and safety of BCD-022, trastuzumab biosimilar candidate, compared to herceptin: results of international multicenter randomized double blind study in patients with HER2+ mBC. ESMO Conference, Copenhagen, Danmark, 2016. Abstract 224.

11. Gligorov J, Curigliano G, Müller V. Assessment of adverse events in patients switching between trastuzumab administration routes (subcutaneous to intravenous and intravenous to subcutaneous) in the PrefHer study. 9th European Breast Cancer Conference, Glasgow, Scotland, 2014. Abstract 229.

12. Jackisch C, Hegg R, Stroyakovskiy D, Ahn JS, Melichar B, Chen SC, Kim SB, Lichinitser M, Starosławska E, Kunz G, Falcon S, Chen ST, Crepelle-Fléchais A, et al. HannaH phase III randomised study: Association of total pathological complete response with event-free survival in HER2-positive early breast cancer treated with neoadjuvantadjuvant trastuzumab after 2 years of treatment-free followup. Eur J Cancer. 2016; 62:62-75.

13. Pivot X, Gligorov J, Müller V, Curigliano G, Knoop A, Verma S, Jenkins V, Scotto N, Osborne S, Fallowfield L. Patients' preferences for subcutaneous trastuzumab versus conventional intravenous infusion for the adjuvant treatment of HER2-positive early breast cancer: final analysis of 488 patients in the international, randomized, two-cohort PrefHer study. Ann Oncol. 2014; 25:1979-1987.

14. North RT, Harvey VJ, Cox LC, Ryan SN. Medical resource utilization for administration of trastuzumab in a New Zealand oncology outpatient setting: a time and motion study. Clinicoecon Outcomes Res. 2015; 7:423-430.

15. De Cock E, Pivot X, Hauser N, Verma S, Kritikou P, Millar D, Knoop A. A time and motion study of subcutaneous versus intravenous trastuzumab in patients with HER2-positive early breast cancer. Cancer Med. 2016; 5:389-397. 